\title{
Endogenous orienting modulates the Simon effect: critical factors in experimental design
}

\author{
Elger L. Abrahamse $\cdot$ Rob H. J. Van der Lubbe
}

Received: 25 August 2006 / Accepted: 6 February 2007 / Published online: 10 March 2007

(c) Springer-Verlag 2007

\begin{abstract}
Responses are faster when the side of stimulus and response correspond than when they do not correspond, even if stimulus location is irrelevant to the task at hand: the correspondence, spatial compatibility effect, or Simon effect. Generally, it is assumed that an automatically generated spatial code is responsible for this effect, but the precise mechanism underlying the formation of this code is still under dispute. Two major alternatives have been proposed: the referential-coding account, which can be subdivided into a static version and an attention-centered version, and the attention-shift account. These accounts hold clear-cut predictions for attentional cuing experiments. The former would assume a Simon effect irrespective of attentional cuing in its static version, whereas the attentioncentered version of the referential-coding account and the attention-shift account would predict a decreased Simon effect on validly as opposed to invalidly cued trials. However, results from previous studies are equivocal to the effects of attentional cuing on the Simon effect. We argue here that attentional cueing reliably modulates the Simon effect if some crucial experimental conditions, mostly relevant for optimizing attentional allocation, are met. Furthermore, we propose that the Simon effect may be better understood within the perspective of supra-modal spatial attention, thereby providing an explanation for observed discrepancies in the literature.
\end{abstract}

E. L. Abrahamse · R. H. J. Van der Lubbe $(\square)$

Cognitive Psychology and Ergonomics,

Faculty of Behavioral Sciences,

Universiteit Twente, Postbus 217,

7500 AE Enschede, The Netherlands

e-mail: R.H.J.vanderLubbe@gw.utwente.nl

\section{Introduction}

It has been repeatedly shown that responses in choice reaction time tasks are slower for contralateral mappings between stimulus and response location than for ipsilateral mappings, even when stimulus location is irrelevant to the task at hand (e.g. Simon \& Rudell, 1967). This observation is known as the Simon effect, the spatial stimulus-response compatibility effect, or the correspondence effect (for reviews, see Simon, 1990; Lu \& Proctor, 1995; Stoffer \& Umiltà, 1997). Most accounts of the Simon effect share the assumption that a spatial code is automatically generated in relation to the irrelevant location of the target stimulus. This code may be directly related to a stimulus, but it may also reflect a supra-modal spatial representation, independent from but linked with various stimulus and also response modalities (e.g. see Van der Lubbe, Jaśkowski, \& Verleger, 2005). Outline of the current paper is to list some experimental conditions that are essential in finding a reliable modulation of attentional cuing on the Simon effect. These conditions can mostly be traced back to factors that play an important role in the allocation of spatial attention, or to implications from recent insights on supramodal spatial attention (in line with variants of the premotor theory of attention; e.g. Rizzolatti, Riggio, Dascola, \& Umiltà, 1987).

The Simon effect has been subject to a long history of debate, and its underlying mechanisms are still under dispute. To explain the effect, Simon (1969) proposed that there is "a natural tendency to respond towards the source of stimulation" (p. 174). Stoffer and Yakin (1994) referred to the possibility that Simon had an attentional explanation in mind, as they stated that reacting towards the source of stimulation is likely to be accompanied by a reflexive shift of attention. Since then, various accounts have related the Simon effect to spatial attention (e.g. Verfaellie, Bowers, \& 
Heilman, 1988a; 1990; Buhlmann \& Wascher, 2006). Based on the idea of spatial coding first put forward by Wallace (1971), Stoffer (1991) and Umiltà and Nicoletti (1992) developed the attention-shift hypothesis, which is one of the major current accounts on the Simon effect. This account is usually contrasted with the referential-coding account.

The attention-shift hypothesis holds that a spatial code is generated through the shift of attention to the location of the imperative stimulus. Shifting the attentional focus to the left or right somehow produces spatial codes that might facilitate left- or right-hand responses, respectively. This implies that the shift of attention immediately preceding the presentation of the imperative stimulus is mainly responsible for the direction of the Simon effect, and that a spatial code for the stimulus is not formed when attentional shifting is prevented (Notebaert, Soetens, \& Melis, 2001; Rubichi, Nicoletti, Iani, \& Umiltà, 1997; Nicoletti \& Umiltà, 1994; Umiltà \& Liotti, 1987). In their study, Nicoletti and Umiltà (1994) briefly presented a letter at fixation, simultaneously with the imperative stimulus (Experiment 2). They reasoned that attention had no time to shift to the stimulus because it had to be kept at fixation, where a letter could signal a catch trial. Consistent with the prediction of the attention-shift hypothesis, no Simon effect was found even though the target appeared to the left or right from fixation. ${ }^{1}$ In a related study by Rubichi et al. (1997), targets were presented to the left or right of the point of fixation, followed by go/no-go stimuli at different positions. The latter indicated whether a response should be executed or withheld. A Simon effect was found that depended on the position of the go-stimulus relative to the target, and not on the position of the target relative to the point of fixation. However, as attention was likely to be directed at the go-stimulus when it was presented, the go-stimulus may be considered as another target signalling the selection of a response referenced to the target position. Further support for the attention-shift hypothesis was presented by Notebaert et al. (2001). They examined the influence of attention shifts by means of a sequential analysis of the stimulus location in a serial reaction-time task. Based on their results from four experiments it was argued that the direction of the shift towards the stimulus caused the Simon effect, rather than the relation of the stimulus to a referent.

According to the other major account, the referentialcoding hypothesis, a spatial stimulus code is derived relative to an intentionally defined object or frame of reference

\footnotetext{
${ }^{1}$ Nevertheless, the idea that attention would not be directed at the target seems somewhat unlikely given the proposed role for attention to select for action (Allport, 1987; Neumann, 1987; Van der Heijden, 1992). Moreover, the question may be raised whether the letter below fixation should not be considered as imperative.
}

(Hommel, 1993). Although Hommel (1993) did not deny that there may be a shift of attention from the intentionally defined object, this is not considered to be sufficient to produce the Simon effect (see also Ivanoff \& Peters, 2000). Further support for the referential-coding account comes from Hommel and Lippa (1995). In their second experiment, they presented the imperative stimuli in the context of a face (left or right eye). Across many face orientations (from a $90^{\circ}$ tilt to the left to a $90^{\circ}$ tilt to the right) the Simon effect occurred within the context of the face, irrespective of the tilt (at a $90^{\circ}$ tilt both eyes were vertically outlined, which would predict no Simon effect from the attention based accounts as only vertical attentional movements are needed). In this version of the referential-coding hypothesis, which is sometimes referred to as the static version (Rubichi et al., 1997; Van der Lubbe \& Woestenburg, 1999), attention is merely an epiphenomenon rather than an essential ingredient for the occurrence of the Simon effect. However, an alternative version of the referential-coding hypothesis has been formulated, proposing the locus of attention as (one of) the point(s) of reference (Umiltà \& Liotti, 1987; Nicoletti \& Umiltà, 1989). This attention-centered version of the referential-coding hypothesis (and sometimes referred to as the dynamic version) incorporates results from a number of studies that showed a modulation of the Simon effect by attentional cueing, both through centrally and peripherally presented cues (e.g. Stoffer \& Yakin, 1994).

The role of attention in the occurrence of the Simon effect has mostly been tested with spatial precuing tasks. In such tasks participants are instructed to keep their eyes fixed at a centrally presented fixation point until target presentation. Attention is then redirected to one of the possible target positions through a spatial precue. These precues can be divided into two types (central and peripheral), which according to several authors recruit partially different attentional mechanisms (Jonides, 1981; Klein, 1994; Posner, 1980). Central (or symbolic) precue stimuli indicate the likely location of the forthcoming target, and are thought to activate endogenous orienting mechanisms that are voluntary and slow. Peripheral onset stimuli are thought to attract attention in a reflexive, fast and involuntary way, irrespective of their informative value (exogenous orienting). After attention is presumably redirected through the spatial cue, the imperative stimulus is presented. If the imperative stimulus appears at the cued location, attention is thought to be focused on target location already (rendering an attentional shift no longer necessary). However, if the imperative stimulus appears on the opposite location, redirecting attention would be required to select and determine a response to the target.

In line with previous studies (Stoffer \& Yakin, 1994; Wascher \& Wolber, 2004), we depicted the following 
Table 1 Peripheral cuing studies that employed cue-target onset intervals up to $1,000 \mathrm{~ms}$

\begin{tabular}{|c|c|c|c|c|c|c|c|c|c|}
\hline Study & $\begin{array}{l}\text { Cor } \times \\
\text { Cue } \\
(\mathrm{RT})\end{array}$ & $\begin{array}{l}\text { Cor } \times \\
\text { Cue } \\
(\mathrm{PC})\end{array}$ & Cue (RT) & Cue (PC) & SOA (ms) & $\begin{array}{l}\text { Target } \\
\text { duration } \\
(\mathrm{ms})\end{array}$ & $\begin{array}{l}\text { Cue } \\
\text { validity } \\
(\%)\end{array}$ & $\begin{array}{l}\text { Control } \\
\text { trials }\end{array}$ & $\begin{array}{l}\text { Eye } \\
\text { control }\end{array}$ \\
\hline Hommel (1993; Exp 4) & No & No & No & Yes & 400 & 150 & 50 & Invalid & No \\
\hline Hommel (1993; Exp 5) & No & No & Yes $(8 \mathrm{~ms})$ & No & 100 & 150 & 50 & Invalid & No \\
\hline Hommel (1993; Exp 6) & No & No & Yes $(8 \mathrm{~ms})$ & No & 50 & 150 & 50 & Invalid & No \\
\hline Stoffer \& Yakin (1994; Exp 1) & Yes & Yes & Yes (60 ms) & No & 133 or 500 ; blocked & 67 & 100 & Neutral & No \\
\hline Zimba \& Brito (1995; Exp 2) & No & No & Yes (29 ms) & No & -50 to 1,000 & 1,000 & 80 & Invalid & Yes \\
\hline Zimba \& Brito (1995; Exp 4) & No & No & Yes (32 ms) & No & 50 to 500 & 1,000 & 80 & Invalid & Yes \\
\hline Van der Lubbe et al. (1996) & Yes & No & Yes (19 ms) & No & 200 & 1,500 & 100 & Neutral & Yes \\
\hline Lupiáñez \& Solano (1997) & No & No & Yes (17 ms) & No & 100 or 1,$000 ;$ mixed & 33 & 50 & Invalid & No \\
\hline Lupiáñez et al. (1997; Exp 1B) & No & No & Yes (40 ms) & Yes & 100 or $400 ;$ mixed & 33 & 50 & Invalid & No \\
\hline $\begin{array}{l}\text { Van der Lubbe \& } \\
\text { Woestenburg (1999) }\end{array}$ & Yes & No & Yes & No & 100 to 300 & 750 & 100 & Neutral & Yes \\
\hline Lupiáñez \& Milliken (1999; Exp 2) & No & No & Yes (15 ms) & Yes & 100 or $400 ;$ mixed & 33 & 100 & Neutral & No \\
\hline $\begin{array}{l}\text { Van der Lubbe \& } \\
\text { Van der Helden (2006) }\end{array}$ & Yes & No & Yes (27 ms) & No & 200 & 100 & 50 & Invalid & Yes \\
\hline
\end{tabular}

SOA stimulus onset asynchrony, Cor difference between correspondence and non-correspondence trials, Cue effect of attentional modulation, either by comparing validly cued trials with invalidly cued trials (cue and targets appeared at opposite sides), or by comparing validly cued trials with neutrally cued trials (see control trials)

predictions for the effect of attentional cuing on the Simon effect. The static version of the referential-coding hypothesis would predict a Simon effect irrespective of the validity of the spatial precue. According to this account, the reference is unaffected by attentional reorientation, and thus remains the same after both valid and invalid cues. Conversely, the attention-centered version of the referentialcoding hypothesis and the attentional-shift hypothesis would both predict the occurrence of a Simon effect mainly in invalidly cued trials. The former holds that references are aligned to the cued location (the locus of attention), rendering a spatially neutral code (or simply no code) for cued targets. From the latter view, target presentation is no longer followed by an attentional shift in validly cued trials, thereby preventing the production of a new spatial code.

Despite these clear-cut predictions, results from previous studies are equivocal to the effects of attentional cuing on the Simon effect. Both with peripheral and symbolic cues, only a few studies found attentional cuing to modulate the Simon effect, whereas many others did not. In the following, we will present an overview of these experiments (see Tables 1, 2). Furthermore, we will list some experimental conditions that we believe are crucial to reliably modulate the Simon effect by attentional cuing. However, because peripheral precues have been found to produce not only an orienting effect but also an alertness effect as well as response tendencies to subsequently presented target stimuli (Van der Lubbe, Keuss, \& Stoffels, 1996; Taylor \& Klein, 1998; Van der Lubbe, Havik, Bekker, \& Postma, 2006a), our major focus will be on symbolic cuing, which may offer a cleaner, purer and more reliable way of assessing the effect of attentional cuing on the Simon effect.

In our overview regarding peripheral cuing studies (see Table 1), we restricted ourselves to those exploiting cuetarget intervals up to 1,000 ms. Wascher and Wolber (2004) proposed that the main difference between peripheral cuing tasks showing a reduction of the Simon effect in validly cued trials and those that do not, concerns the validity of cue information. Specifically, studies employing $100 \%$ cue validity (Stoffer \& Yakin, 1994; Umiltà \& Liotti, 1987; Van der Lubbe \& Woestenburg, 1999) showed a reduction in the Simon effect, whereas studies that used lower cue validities (e.g. Zimba \& Brito, 1995; Lupiáñez, Milan, Tornay, Madrid, \& Tudela, 1997) showed no change of the Simon effect $^{2}$ due to attentional precuing. Nevertheless, their statement was recently challenged by a study of Van der Lubbe and Van der Helden (2006), in which a clear modulation of the Simon effect was found by uninformative precues: a larger Simon effect for invalidly cued trials. Another explanation for the observed discrepancies may be unreliability of the effects of the precues themselves. As mentioned above, studies using peripheral precues to orient attention are presumably affected by response tendencies, which may

\footnotetext{
${ }^{2}$ These studies compared the occurrence of the Simon effect between validly cued trials, and trials with a neutral (temporal) cue or no cue. As cues were $100 \%$ valid, these cues were also predictive. Therefore, these differences might be specific to the involvement of endogenous orienting.
} 


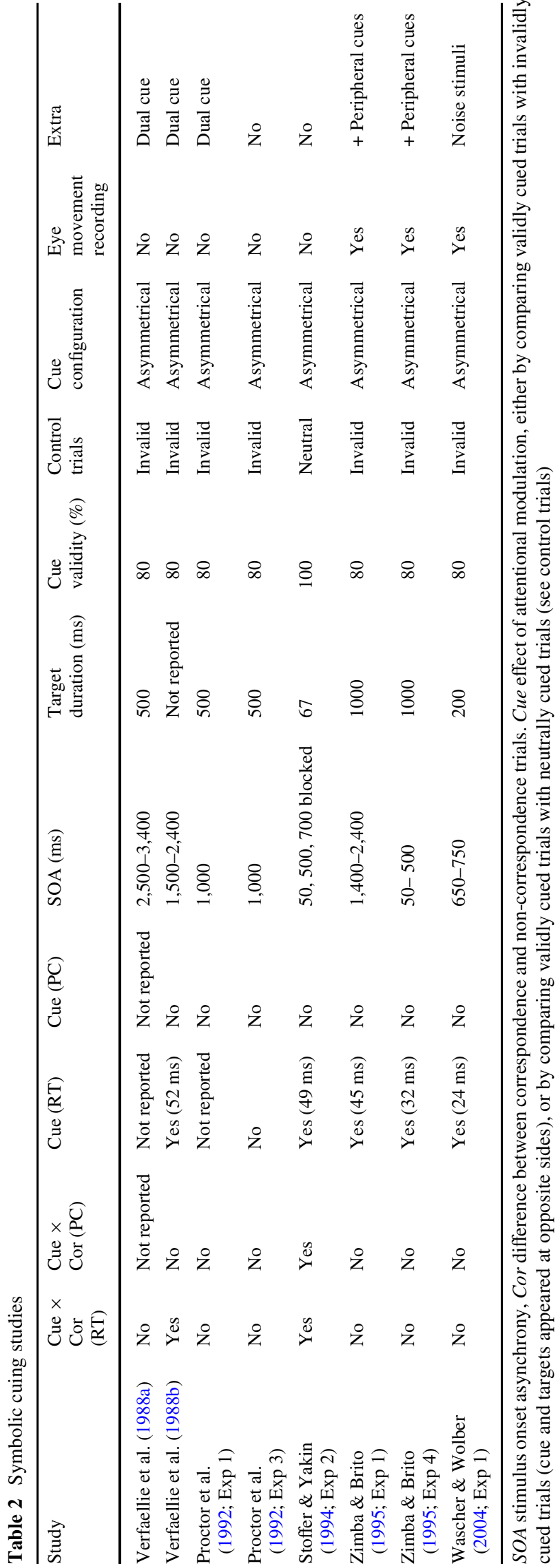

have a profound effect on the task at hand, thereby rendering results unpredictable and difficult to interpret.

A more reliable way of experimentally controlling the effect of attentional orienting within a certain task appears the employment of spatially informative symbolic cues. As far as we know, the only additional effect besides the orienting of attention was depicted by Eimer (1995), who stated that using arrows as central cues can automatically induce motor activation. This can easily be overcome by introducing stimuli that equally prime both sides by their sole configuration as central cues. We will return to this topic below. Like the peripheral cuing studies, those that employed symbolic precues have not produced the univocal results that one would expect from the clear predictions that are set by the two major accounts on the Simon effect, either. Only a few studies have found symbolic cuing to modulate the Simon effect (Verfaellie, Bowers, \& Heilman, 1988b; Stoffer \& Yakin, 1994), but most of them showed a Simon effect irrespective of the spatial cues (see Table 2). Verfaellie et al. (1988b) independently manipulated two components-selective attention and intention-in a dual cue design. The Simon effect was much smaller on trials with attentional cues than on trials without attentional cues, supporting an attention-based account of the Simon effect. However, their results have not been replicated in two attempts. In Verfaellie et al. (1988a) a more or less comparable setting was used as in Verfaellie et al. (1988b). Again, they found no Simon effect on trials with only attentional cues. However, this time no Simon effect was found either on those trials that contained no precue at all. Finally, Proctor, Lu, and Van Zandt (1992) performed a close procedural replication of Verfaellie et al. (1988a). They found a Simon effect irrespective of the attentional precues. Thus, from all the relevant studies we know, the only one unambiguously showing a clear modulation of symbolic attentional cuing on the Simon effect is Stoffer and Yakin (1994). It appears, then, that the interaction between the Simon effect and attentional cuing is rather subtle and sensitive to experimental conditions.

Below some critical recommendations are listed that are thought to optimize the experimental conditions necessary for finding a reliable and replicable modulation of the Simon effect by symbolic cuing. These can be subdivided in factors that may be critical in optimizing the allocation of spatial attention to prevent type II errors (variability of cuetarget interval and cue complexity), implications from a supramodal perspective of spatial attention (target duration), and some other minor issues.

First of all, most of the relevant studies exploited variable cue-target intervals (see Table 2). We recognize that typical spatial cuing tasks (e.g. Posner, 1980; Posner \& Cohen, 1984) usually employ variable cue-target intervals, but the reason for this choice is mostly to study changes in 
attentional allocation over time rather than optimizing attentional allocation. However, as optimizing the allocation of spatial attention is crucial in the current design, we believe that variable intervals may have a negative affect on the task at hand. The allocation of attention might be automatically influenced by the cue-target interval from the former trial (Jongen \& Smulders, 2006; Van der Lubbe, Los, Jaśkowski, \& Verleger, 2004), meaning that the temporal anticipation to the target in the current trial (through the allocation of attention) can be disturbed if the cue-target interval is variable. Furthermore, by creating temporal uncertainty, information about time cannot be used for the optimal deployment of attentional resources (Miniussi, Wilding, Coull, \& Nobre, 1999). A more or less fixed cuetarget interval, then, seems to be an important condition to optimize the potential effect of attentional cueing on the Simon effect. Furthermore, the cue-target interval must be within a range that permits participants to effectively focus their attention, without loosing their focus because of too lengthy intervals.

Second, some of the previous studies employed a dual cue design (i.e. intentional and attentional precues; Verfaellie et al., 1988a, b; Proctor et al., 1992). In these studies, intentional and attentional precues were presented above or below the central fixation point (counterbalanced across subjects). The direction of the two cues could be either compatible with each other or not. This may well have had a detrimental effect on the allocation of spatial attention. First of all, besides indicating the correct response, intentional precues are likely to induce attentional orienting as well, either to the relevant response button, the required hand, or both. This effect might especially interfere with effects of the attentional precue when both cues are incompatible. Second, because of the complexity of instructions, subjects have to divide their attentional resources, which may imply that fewer resources are available to focus on the likely target position as compared to a single attentional cuing design. To reduce complexity, participants may employ a strategy in which they follow the attentional cue on a specific proportion of trials, and the intentional cue on another proportion of trials, which would result in a suboptimal setting.

Third, the duration of the target presentation is usually within the range of 500-1,000 ms. This long duration is likely to provoke preparation of an eye movement towards the relevant side. As will be elaborated in the general discussion, spatial codes might be generated (and interfere with response selection) at several moments in time, and through several closely related mechanisms. More specifically, it could be that, even though attention stays focused on the cued position, preparing an eye movement produces a new spatial code in line with variants of the premotor theory of attention (Rizzolatti et al., 1987; Eimer, Forster, Van
Velzen, \& Prabhu, 2005; Van der Lubbe, Neggers, Verleger, \& Kenemans, 2006b). ${ }^{3}$ Shorter durations of the target presentation may reduce the intention to prepare and execute an eye movement towards the relevant side, and therefore prevent the generation of interfering spatial codes (as can be expected from the attentional-shift perspective).

Fourth, there are some minor issues to consider that may unintentionally affect performance, and interfere with the experimental design. Many studies that examined the effect of endogenous attentional cueing on the Simon effect used arrows as central cues (e.g. Verfaellie et al., 1988a). Eimer (1995) stated that when stimuli and responses overlap with respect to spatial attributes, automatic response activation processes are triggered. This S-R-compatibility might produce Simon-like effects, rendering the data ambiguous with regard to the underlying theoretical explanations. Another issue about the experimental design concerns the cueing conditions. Some studies assessed the effect of attentional cueing by comparing validly cued trials with neutrally cued trials. It may well be that in neutrally cued trials attention is divided across both potential target positions. At the presentation of the target, then, attentional zooming may be performed instead of attentional shifting (Stoffer, 1991). Therefore, a more appropriate way appears to be a comparison of validly and invalidly cued trials.

Finally, many of the relevant studies on the topic did not check for the possibility that the attentional shift was accompanied by movements of the eyes (e.g. Stoffer \& Yakin, 1994; Proctor et al., 1992; Verfaellie et al., 1988a, b). Even though subjects are instructed to keep their eyes at the central fixation point during trial execution, they usually have trouble repressing an eye movement. Without the guaranteed exclusion of eye movements, the validity of ascribing effects to the manipulation of attention would be lost as the relevant stimulus location may be used as the new reference after reorientation of eye focus.

There are some studies that seem to meet these recommendations more or less (Proctor et al., 1992; Stoffer \& Yakin, 1994; Wascher \& Wolber, 2004; see Tables 1, 2). Stoffer and Yakin (1994) showed a significant modulation of the Simon effect by symbolic attentional cuing. They used three different SOAs (50, 500 and $700 \mathrm{~ms})$, but these were fixed within blocks. Furthermore, they presented the imperative stimulus for only $67 \mathrm{~ms}$. Importantly, the benefit of precuing in the form of a reduction of the size of the Simon effect was smallest in the 50-ms-SOA condition and

\footnotetext{
${ }^{3}$ This theory proposes that attentional orienting might be identical to the planning of saccades (Rizzolatti et al., 1987), which states that attentional orienting might be equated with the preparation of actions in general (Eimer et al., 2005), or that there exists functional overlap between attentional orienting and saccade planning (Van der Lubbe et al., 2006b).
} 
Fig. 1 An example of the stimuli and their temporal order as employed in Experiment 1 and 2

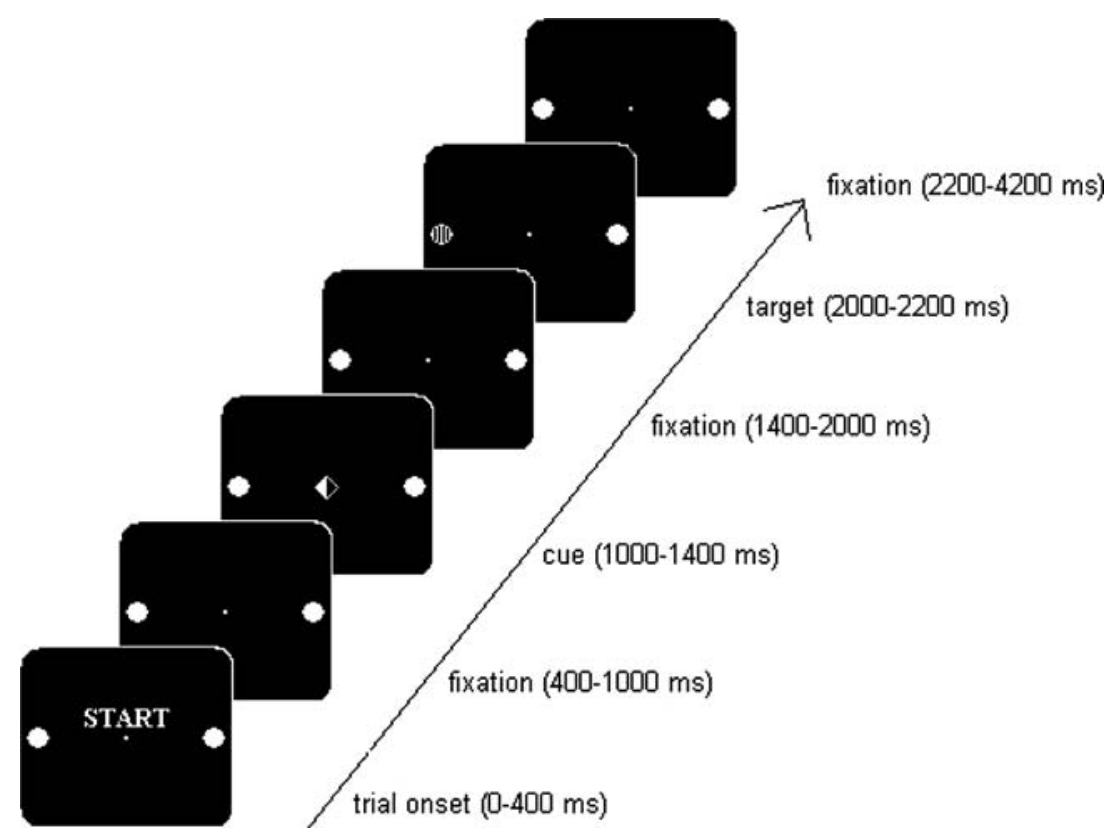

by far the largest in the 700-ms-SOA condition. This suggests that a substantial reduction of the Simon effect can be expected only when the refocusing of attention from fixation to the imperative stimulus can be completed before presentation of the imperative stimulus. This strengthens the idea of an attention-based account. Wascher and Wolber (2004) used slightly variable SOAs (between 650 and $750 \mathrm{~ms}$ ) and presented target stimuli for $200 \mathrm{~ms}$. Even though this matches most of our recommendations, they did not find a modulation of the Simon effect due to symbolic cuing. However, simultaneously with the target presentation they presented a noise stimulus at the opposite stimulus location (primarily to avoid exogenous asymmetries in the EEG; Praamstra \& Plat, 2001). This additional stimulus may not essentially affect the characteristics of the Simon effect (or even enhance the Simon effect; Proctor \& Lu, 1994), but in our view may exert a detrimental effect on the effectiveness of attentional orienting. It has been demonstrated several times that peripheral-visual onset cues (to which the noise stimuli are essentially equivalent) attract spatial attention even if these distracting stimuli are without any informational value and the subjects are explicitly instructed to ignore them (Lambert \& Hockey, 1991; Maylor, 1985; Maylor \& Hockey, 1987; Posner \& Cohen, 1984). In their study, Wascher and Wolber (2004) do not find a large main effect of attentional cuing in the first place, leaving no or little room for a significant interaction with the Simon effect. This is even more true for Proctor et al. (1992). In their study (Experiment 3), they reported an insignificant main effect of attentional cuing. However, how can one expect to find a modulation of attentional cuing on the Simon effect when attention itself is ineffectively manipulated?
In the current study, the experimental conditions for observing a modulation of the Simon effect due to attentional orienting were optimal with regard to the above considerations (see Fig. 1). EOG was recorded in order to exclude trials in which eye movements were made during critical time intervals. The cue-target interval was set at $1,000 \mathrm{~ms}$ for every trial, as to minimize sequential effects and to reduce temporal uncertainty. This should be long enough for participants to focus their attention on the cued location. Target presentation duration was restricted to $200 \mathrm{~ms}$ to discourage participants to prepare eye movements just before responding. Furthermore, invalidly cued trials were employed rather than neutral cues, while the spatial cues consisted of two diamond shaped figures pointing in opposite directions. The latter was done as to prime both spatial locations equally by the sole configuration of the cue.

In line with the attention-centered version of the referential-coding hypothesis and the attentional-shift hypothesis we predicted the Simon effect to be largest on invalidly and smallest on validly cued trials. From the perspective of the referential-coding hypothesis, a reference is intentionally defined at the start of each trial. This could be either the central fixation point (which is most probable because the eyes are always fixated there), or one of the two circles that define possible stimulus locations (see Fig. 1). Throughout a trial this reference may change due to the onset of a new stimulus (in our study only the centrally located cue), due to an eye movement (eye-centered coding), or an attention movement (attention-centered coding). Our predictions in the current design are similar to those employed in previous studies (e.g. Stoffer \& Yakin, 1994; Wascher \& Wolber, 2004). 


\section{Experiment 1}

Method

\section{Participants}

Informed consent was obtained from 14 participants, mostly students of Utrecht University. Three participants were excluded from the analyses because of too many eye movements $(>50 \%)$ during critical time intervals. The remaining 11 participants (mean age 24 years, 9 right- and 2 left-handed) had normal or corrected to normal vision and intact colour vision, were in good physical health, and had no history of psychiatric or neurological disorder. Participants received $€ 45$ for their participation, of which the current experiment was only a part (see Van der Lubbe et al., 2006b). The study was approved by the local ethics committee of the faculty of social sciences of Utrecht University.

\section{Stimulus, apparatus and recording}

All stimuli were presented on a black computer screen (see Fig. 1). During each trial, a light-grey fixation dot $\left(0.2^{\circ} \times 0.2^{\circ}\right)$ was continuously presented in the centre of the screen, accompanied by two light-grey open circles $\left(r=0.34^{\circ}\right)$ located $8.3^{\circ}$ to the left and right of the fixation dot (default-display). Trials started when the word "START" was displayed $0.2^{\circ}$ above the fixation dot. The display with the word lasted for $400 \mathrm{~ms}$, after which the default-display was presented again for $600 \mathrm{~ms}$. Next, the cue was presented in the centre, replacing the fixation dot for a duration of $400 \mathrm{~ms}$. The cue was a diamond (height $0.85^{\circ}$, width $1.71^{\circ}$ ) constructed of a green and a red triangle, each pointing to one of the circles. After the cue, the default-display was presented again for $600 \mathrm{~ms}$. Thus, the preparatory interval from cue onset to target onset amounted to $1,000 \mathrm{~ms}$. Next the target was displayed within one of the circles for a duration of $200 \mathrm{~ms}$, consisting of either three horizontal or vertical lines. After target offset, the default-display was presented for another $2,000 \mathrm{~ms}$.

Participants were seated in a comfortable armchair in a silenced and darkened chamber, in front of a $17^{\prime \prime}$ screen monitor (DELL) at a distance of $100 \mathrm{~cm}$. Presentation software (version 0.43 developed by Neurobehavioral Systems) was used for stimulus presentation and the production of external triggers. The external triggers were received by Vision Recorder (version $1.0 \mathrm{~b}$ BrainProducts $\mathrm{GmbH}$ ) which measured participants' EOG (electrooculography), EEG (electroencephalogram, reported in Van der Lubbe et al., 2006b) and their button presses. The buttons were fixed in two response boxes, which were placed in a com- fortable position at the left and right side on a hand-rest in front of the participant, approximately $25 \mathrm{~cm}$ apart.

EOG was measured above and below the left eye, and horizontally from the outer canthi of both eyes to determine the vEOG and the hEOG. EOG was amplified by a BrainAmp amplifier (BrainProducts $\mathrm{GmbH}$ ), and was recorded at $250 \mathrm{~Hz}$ and digitally filtered $(\mathrm{TC}=5.0 \mathrm{~s}$, low-pass filter of $100 \mathrm{~Hz}$, notch filter of $50 \mathrm{~Hz}$ ) by Vision Recorder. Electrode resistance was kept below $5 \mathrm{k} \Omega$.

\section{Task and procedure}

Participants performed a 640 trial choice-response task, lasting for approximately $45 \mathrm{~min}$. Each task was divided into two parts. In the first part, half of the participants were informed that the circle indicated by the green side of the cue was the most probable target location (on $80 \%$ of the trials, i.e. the valid trials). On $15 \%$ of the trials, the target occurred in the other circle (invalid trials), whereas on 5\% of the trials, no target occurred (catch trials). In the second part, they were informed that the red side of the cue indicated the most probable target location. For the other half of the participants, this order was reversed. The trials were divided into four blocks of 160 trials, which were each preceded by 20 practice trials. Participants were instructed to keep their eyes on the fixation dot during the cue-target interval, and to press the left or right button when one of the circles was filled with horizontal or vertical lines, respectively. Button presses had to be as fast and accurate as possible. As target position and the required button press varied independently, target position and response side could correspond or not (corresponding vs. non-corresponding trials). The latter factor was not included in the earlier analyses reported by Van der Lubbe et al. (2006b).

\section{Results}

Trials with detectable lateral eye movements (exceeding $60 \mathrm{~V}$ in the hEOG recording) from cue onset until target onset were removed from analyses, which left $93.4 \%$ of the trials. Reaction time (RT) was measured relative to target onset. There were no responses faster than $100 \mathrm{~ms}$ (premature), and responses slower than $1,500 \mathrm{~ms}$ (misses) and erroneous responses (incorrects) were excluded from proportions of correct responses (PCs). Mean RTs and PCs as a function of correspondence and cue validity are compiled in Table 3. Correspondence effects on RT and PC are displayed in Fig. 2.

A repeated measures ANOVA was performed on RT with correspondence (corresponding vs. non-corresponding trials) and cue validity (valid vs. invalid trials) as withinsubject variables. This revealed significant main effects of correspondence, $F(1,10)=14.0, P<0.005$, and of cue 
Table 3 Mean RT (in ms) and PC (in \%) and their standard errors (in between brackets) for corresponding (Corr) and non-corresponding trials (Nonc) in case of valid and invalid symbolic cues in Experiments 1 and 2

\begin{tabular}{|c|c|c|c|c|c|c|c|c|}
\hline & \multicolumn{4}{|c|}{ Reaction times } & \multicolumn{4}{|c|}{ Proportion correct } \\
\hline & \multicolumn{2}{|l|}{ Valid } & \multicolumn{2}{|l|}{ Invalid } & \multicolumn{2}{|l|}{ Valid } & \multicolumn{2}{|l|}{ Invalid } \\
\hline & Corr & Nonc & Corr & Nonc & Corr & Nonc & Corr & Nonc \\
\hline Experiment 1 & $714(37)$ & $735(37)$ & $788(32)$ & $842(42)$ & $95.2(1.4)$ & $92.6(2.4)$ & $91.3(3.0)$ & $86.9(4.2)$ \\
\hline Experiment 2 & $631(43)$ & $643(38)$ & $683(50)$ & $721(43)$ & $95.0(2.1)$ & $93.6(1.6)$ & $91.6(2.8)$ & $89.6(2.2)$ \\
\hline
\end{tabular}

Fig. 2 The correspondence or Simon effect on RT (in ms) and PC (in \%) for validly and invalidly cued trials in Experiments 1 and 2. Corresponding and noncorresponding trials are abbreviated as corr and nonc. Hence, nonc-corr on RT reflects a positive Simon effect (i.e. faster responses for corresponding than for non-corresponding trials), whereas corr-nonc on PC reflects more accurate performance on corresponding than on noncorresponding trials. Note that the values for RT and PC are indicated at the left and right vertical axes, respectively

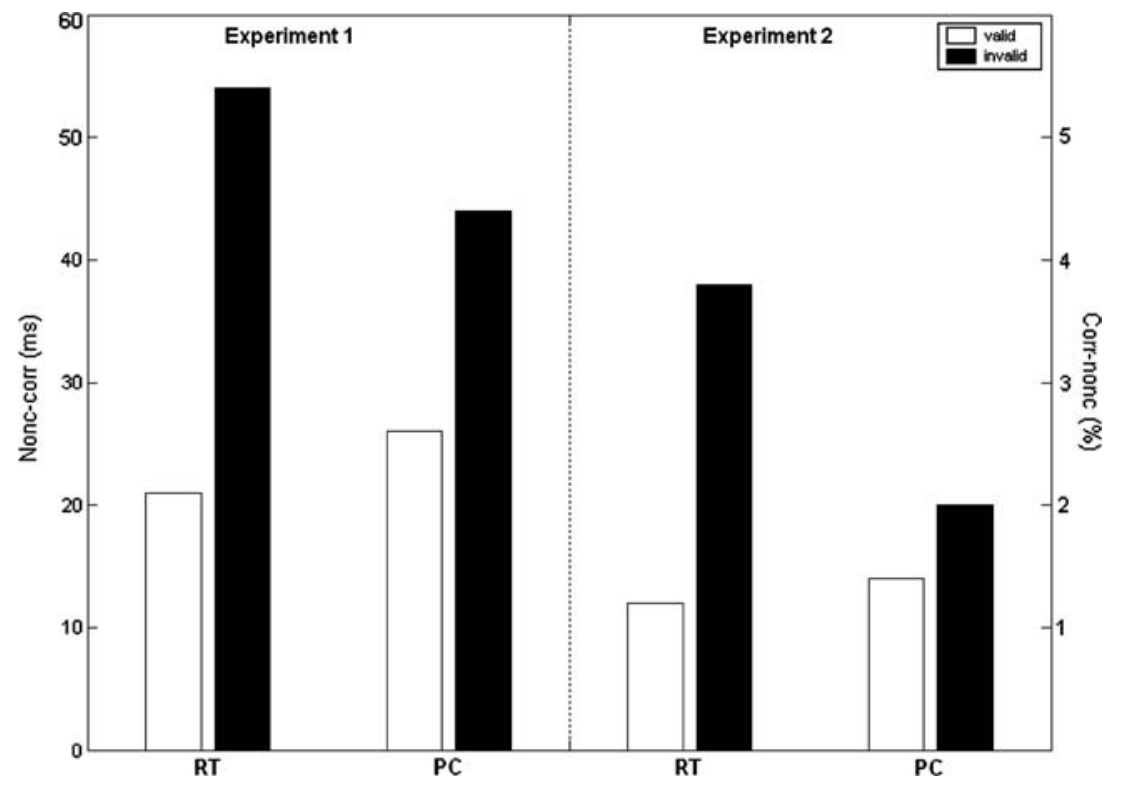

Discussion

The results from Experiment 1 indicate that redirecting attention shortly after target presentation enhances the Simon effect. In line with the attentional shift hypothesis and the attention-centered version of the referential-coding account, this suggests that attention plays an important role in the occurrence of the Simon effect. This issue will be elaborated on in the general discussion. As many previous studies failed to find a significant interaction between correspondence and cue validity (see Tables 1,2 ), we decided to include a replication of Experiment 1. The lack of consistency and reliability among previous studies (e.g. Verfaellie et al., 1988a, b) indeed motivates concerns about a possible type I error in Experiment 1.

\section{Experiment 2}

Method

Most aspects were the same as in Experiment 1, as Experiment 2 was an exact duplicate. The only relevant changes concerned the participants involved, the moment of carrying 
out the experiment ( 3 years later), and the experimenter involved. Informed consent was obtained from 18 participants, but eight participants had to be removed from the analyses, either because of too many eye movements during critical time intervals, or because of procedural errors (five), which left ten participants (mean age 21.4 years, nine right- and one left-handed).

\section{Results and discussion}

Trials with detectable lateral eye movements from cue onset until target onset were excluded from the analyses. This left on average $82.1 \%$ of the data. Mean RTs and PCs as a function of correspondence and cue validity are compiled in Table 3, and correspondence effects on RT and PC are displayed in Fig. 2.

A repeated measures ANOVA was performed on RTs with correspondence and cue validity as within-subject variables. This revealed a significant main effect for cue validity, $F(1,9)=25.5, P<0.005$, but not for correspondence ( $25 \mathrm{~ms}), F(1,9)=4.7, P<0.06$. Again, this indicates that validly cued trials are responded to faster than invalidly cues trials (the cuing effect amounting to $66 \mathrm{~ms}$ ). Most importantly, a significant interaction was found for correspondence and cue validity, $F(1,9)=6.6, P<0.05$. Separate paired sample $t$ tests revealed a difference between corresponding and non-corresponding trials for invalid cueing, $t(9)=2.7, P<0.05$, but not for valid cueing. These findings suggest that within the current experimental setup, attentional cueing has a rather robust modulatory effect on the size of the Simon effect.

A second repeated measures ANOVA was run on PCs with correspondence and cue validity as within-subject variables. This revealed a significant effect of cue validity, $F(1,9)=19.0, P<0.01$, but neither an effect of correspondence nor an interaction between correspondence and cue validity. Participants produced more errors in trials with invalid spatial cueing than with valid spatial cueing (9.4 vs. $5.7 \%)$. The difference between correspondence and noncorrespondence trials for validly and invalidly cued trials amounted to 1.4 and $2.0 \%$ respectively, signifying that effects on RT cannot be attributed to speed-accuracy tradeoff.

Finally, an omnibus analysis was performed on both experiments for the sake of completeness. A repeated measures ANOVA was run on RTs with correspondence and cue validity as within-subject variables, and Experiment as between-subject variable. This resulted in significant main effects of correspondence, $F(1,19)=16.9, P<0.005$, and cue validity, $F(1,19)=84.1, P<0.001$. The correspondence $\times$ cue validity interaction was highly significant, $F(1,19)=15.0, P<0.005$, showing a strong modulation of attentional cuing on the Simon effect. Separate paired sample $t$ tests revealed a difference between corresponding and non-corresponding trials for both valid $(16 \mathrm{~ms})$, $t(20)=2.5, P<0.05$, and invalid $(47 \mathrm{~ms})$ cuing, $t(20)=4.7$, $P<0.001$. Importantly, the three-way interaction between correspondence, cue validity and experiment was far from significant, $F(1,19)=0.2$. Another repeated measures ANOVA with the same factors run on PCs resulted in a significant main effect of cue validity, $F(1,19)=10.9$, $P<0.005$. Participants produced more errors in trials with invalid cuing $(10.1 \%)$ than with valid cuing $(5.9 \%)$. All other effects were far from significant $\left(F_{\mathrm{s}}<2.4\right)$.

\section{General discussion}

The current study provides new and convincing support for the involvement of attention in the occurrence of the Simon effect. In both experiments, a significantly smaller Simon effect was obtained for targets at validly cued (attended) locations than at invalidly cued (unattended) locations. The modulation of the Simon effect by attentional cueing can be reliably shown as long as some crucial conditions are met. This has important implications for the presumed mechanisms underlying the Simon effect.

The present findings are in line with the attentional shift hypothesis and the attention-centered version of the referential-coding hypothesis. Both accounts predict that the Simon effect would be manifest mainly under conditions in which the location of the imperative stimulus does not coincide with the location of the focus of attention. The obtained results support this prediction. Conversely, the findings contradict the static version of the reference hypothesis. From this view, each stimulus produces a spatial code relative to the reference, with attention playing no part in the formation at all. In the current study the eyes were always kept at fixation, and no new stimuli were presented other than the central spatial cue. As the reference would therefore remain unchanged from the beginning of each trial, the same spatial codes would be generated for every stimulus, predicting a Simon effect independent of attentional cueing. Clearly, this version falls short of explaining the results of the current study.

As is noted above, the current study is unable to discriminate between the attentional-shift hypothesis and the attention-centered version of the reference hypothesis. However, what seems to be clear is that the occurrence of the Simon effect is attention based: the spatial codes that interfere with response selection seem to be dependent on the locus of attention just before target presentation. These codes may be exclusively related to a stimulus, but may also reflect a supramodal spatial representation. It may indeed be argued that spatial codes responsible for the Simon effect are generated by multiple closely related mechanisms (see also Stoffer \& Yakin, 1994), and on multiple moments in time, 
which may provide an important additional reason why it has been rather difficult to establish a modulation of the Simon effect due to attentional orienting. In the following section we will more deeply focus on arguments supporting this supramodal view, and on its implications.

\section{The Simon effect from the perspective of supramodal spatial attention}

In the previous section we clarified that the locus of attention plays an important role for the Simon effect. It has additionally been shown that the likely moment of attentional selection of a stimulus rather than stimulus onset plays a crucial role for the formation of spatial codes (Van der Lubbe et al., 2005). Hence, spatial attention appears to be an important ingredient of the underlying mechanism responsible for the Simon effect. In the field of spatial attention significant progress has been made, which according to our opinion has important implications for interpreting the Simon effect. First, several studies have revealed that taskirrelevant non-predictive auditory cues in the left or right field preceding visual targets to the left and right, affect the speed and accuracy of responses to these targets (Spence \& Driver, 1997; Schmitt, Postma, \& De Haan, 2000; Van der Lubbe \& Postma, 2005). Namely, responses are faster when auditory cues and visual targets originate from the same location as compared to when they occur at different sides from fixation. The latter findings suggest that spatial representations or maps of the auditory and visual modality are somehow interlinked. Secondly, while preparing a left or right hand movement, a left or right saccade, or attending to a location because of a likely visual, auditory, or tactile target at a specific location, highly comparable brain activations have been observed (Van der Lubbe, Wauschkuhn, Wascher, Niehoff, Kömpf \& Verleger, 2000; Eimer, Van Velzen, \& Driver, 2002, Van der Lubbe et al., 2006b). These findings suggest (for an early advocate of this view see Farah, Wong, Monheit, \& Morrow, 1989) that there exists a kind of supramodal spatial map that interconnects not only visual, auditory and tactile space, but also motor space for hand movements and eye movements. At a neurophysiological level, attending to a specific location may be realized by means of activation of neurons representing a specific part of supramodal space, thereby affecting processing of stimuli and responses concerning a specific location. Physiological support for the likely locus of these different spatial representations in parietal cortex comes from several recent fMRI studies (e.g. Astafiev, Shulman, Stanley, Snyder, Van Essen, \& Corbetta, 2003).

What are possible implications of these interconnected spatial modules for interpreting the Simon effect? Reconsidering the pioneering study of Simon and Craft (1970) in which an irrelevant auditory cue affected the speed of responses towards centrally presented visual targets, this result may actually be interpreted as a crossmodal attention effect, in which spatial maps of hand-motor space and auditory space are interlinked, either directly, or indirectly by means of an intermediate supramodal module. In short, presenting an auditory stimulus to the left may, by means of a supramodal spatial module, activate left hand-motor space, thereby speeding up responses towards that same location. The same multimodal mechanism may account for the observation of a Simon effect with bilateral stimuli, such as in the studies of Wascher and Wauschkuhn (1996) and Van der Lubbe, Jaśkowski, Wauschkuhn, and Verleger (2001). Attentional selection of the relevant stimulus, implemented by the supramodal spatial module, not only enables selection of the relevant stimulus, but also induces activation of corresponding hand-motor space, thereby leading to a Simon effect. Nevertheless, not all findings seem so easy to account for in terms of supramodal attention.

In the study of Van der Lubbe et al. (2005) multiple-item arrays were used and the locus of a target within the array was indicated by a precue occurring before the target, a simultaneous cue presented together with the target, and a postcue occurring after the target. Responses were fastest in the precue condition, but the Simon effect was of comparable size in all conditions. As additionally confirmed by effects on ERPs, attention was apparently allocated to the cued location, which suggests, opposed to the results of the current study, that the Simon effect is unaffected by the locus of attention. However, ERPs additionally revealed that attention was oriented again in direction of the relevant side after target onset, which seems surprising from the perspective of unimodal visual-spatial attention, as attention was already directed at the relevant location (see also Wascher \& Wolber, 2004). However, this second ERP effect might reflect selection of the relevant side occurring as a part of planning an eye movement towards the relevant side (e.g. see Van der Lubbe et al., 2006b). This second activation may be the reason why the Simon effect remained present in the precue condition. Thus, from the perspective of a multimodal mechanism, spatial codes may be generated several times, not only when selecting a target at a specific location, but also when selecting a location for other purposes, such as the execution of a hand- or eyemovement. If the latter type of selection occurs shortly before actual selection of the required response, then interference of this spatial code will be observed. As a consequence, a likely reason why a modulation of the Simon effect was found in the current study but not in the study of Van der Lubbe et al. (2005) is that the production of spatial codes after target selection was less in the current study, due to the shorter presentation duration of the targets. In the study of Van der Lubbe et al. (2005) stimuli remained on the screen until a response was made, making eye movements 
towards the relevant side rather useful. In the current study, the target disappeared after $200 \mathrm{~ms}$, and was also presented without flanking distractors. These beneficial aspects may have reduced the intention to prepare an eye movement towards the relevant side, and therefore, may have resulted in a reduced Simon effect on validly cued trials in the current study.

Additionally, in a study of Van der Lubbe and Van der Helden (2006) it was observed that the Simon effect is modulated by exogenous cues in unimodal settings, but not in crossmodal settings. More specifically, they found only visual precues, and not auditory precues, to modulate the Simon effect with visual targets (it should be noted, however, that there was no significant main cuing effect for the auditory precues). This finding seems to accord with the view that attention plays an important role for the Simon effect, but questions the directness of links between maps of visual and auditory space. So, despite the many studies that support a supramodal perspective on spatial attention, this demonstrates the complexity of the subject of interest.

In conclusion, the current study demonstrates that endogenous orienting modulates the Simon effect, being reduced (or absent) on validly as compared to invalidly cued trials. These findings confirm hypotheses on the Simon effect that either the reference depends on the current focus of attention or that spatial codes are related to attentional shifts. Several methodological reasons like variable cue-target intervals and long presentation times of targets may have been responsible for the presence of null effects in earlier studies. It was argued that a multimodal perspective on spatial attention, in which several perceptual and motor spatial maps are interlinked, may help in understanding the presence or absence of a modulation of the Simon effect. Specifically, the Simon effect was argued to be due to the production of spatial codes, which may not only be activated when selecting a target at a specific location, but also when selecting that location for other purposes, such as the execution of eye-movements.

Acknowledgments The current study was partially supported by a grant from the Netherlands Organization for Fundamental Research to Albert Postma (NWO: 440-20-000). Thanks are due to two anonymous reviewers and Carlo Umiltà for their helpful comments on an earlier draft, and to Willem Holleman and Daan Vroon for their help in measuring the participants.

\section{References}

Allport, D. A. (1987). Selection for action: some behavioral and neurophysiological considerations of attention and action. In H. Heuer, \& A. F. Sanders (Eds.), Perspectives on perception and action. Hillsdale, NJ: Lawrence Erlbaum Associates Inc.

Astafiev, S. V., Shulman, G. L., Stanley, C. M., Snyder, A. Z., Van Essen, D. C., \& Corbetta, M. (2003). Functional organization of human intraparietal and frontal cortex for attending, looking, and pointing. Journal of Neuroscience, 23, 4689-4699.

Buhlman, I., \& Wascher, E. (2006). Intentional pre-cueing does not influence the Simon effect. Psychological Research, 70, 117-124.

Eimer, M. (1995). Stimulus-response compatibility and automatic response activation: Evidence from psychophysiological studies. Journal of Experimental Psychology: Human Perception and Performance, 21, 837-854.

Eimer, M., Van Velzen, J., \& Driver, J. (2002). Cross-modal interactions between audition, touch, and vision in endogenous spatial attention: ERP evidence on preparatory states and sensory modulations. Journal of Cognitive Neuroscience, 14, 254-271.

Eimer, M., Forster, B., Van Velzen, J., \& Prabhu, G. (2005). Covert manual response preparation triggers attentional shifts: ERP evidence for the premotor theory of attention. Neuropsychologia, 43, 957-966.

Farah, M.J., Wong, A.B., Monheit, M.A., \& Morrow, L. (1989). Parietal lobe mechanisms of spatial attention: Modality-specific or supramodal?. Neuropsychologia, 27, 461-470.

Hommel, B. (1993). The role of attention for the Simon effect. Psychological Research, 55, 208-222.

Hommel, B., \& Lippa, Y. (1995). S-R compatibility effects due to context-dependent spatial stimulus coding. Psychonomic Bulletin and Review, 2, 370-374.

Ivanoff, J., \& Peters, M. (2000). A shift of attention may be necessary, but it is not sufficient, for the generation of the Simon effect. Psychological Research, 64, 117-135.

Jongen, E. M. \& Smulders, F. T. (2006). Sequence effects in a spatial cueing task: Endogenous orienting is sensitive to orienting in the preceding trial. Psychological Research, doi:10.1007/s00426006-0065-3.

Jonides, J. (1981). Voluntary vs. automatic control over the mind's eye's movement. In J. Long, \& A. Baddeley (Eds.), Attention and performance VIII (pp. 259-276). Hillsdale, NJ: Erlbaum.

Klein, R. M. (1994). Perceptual-motor expectancies interact with covert visual orienting under conditions of endogenous but not exogenous control. Canadian Journal of Experimental Psychology, 48, 167-181.

Lambert, A., \& Hockey, R. (1991). Peripheral visual changes and spatial attention. Acta Psychologica, 76, 149-163.

Lu, C.-H., \& Proctor, R. W. (1995). The influence of irrelevant local information on performance: A review of the Simon effect and spatial Stroop effects. Psychonomic Bulletin and Review, 2, 174-207.

Lupiáñez, J., Solano, C. (1997). Inhibición de retorno en una tarea de discriminación de color: no interacción con el efecto Simon [Inhibition of return in a color discrimination task: no interaction with the Simon effect] [Abstract in English]. Cognitiva, 9, 195-205.

Lupiáñez, J., \& Milliken, B. (1999). Inhibition of return and the attentional set for integrating versus differentiating information. The Journal of General Psychology, 126, 392-418.

Lupiáñez, J., Milán, E. G., Tornay, F. J., Madrid, E., \& Tudela, P. (1997). Does IOR occur in discrimination tasks? Yes, it does, but later. Perception and Psychophysics, 59, 1241-1254.

Maylor, E. A. (1985). Facilitatory and inhibitory components of orienting in visual space. In M. I. Posner, \& O. S. M. Marin (Eds.), Attention and performance XI (pp. 189-204). Hillsdale, NJ: Lawrence Erlbaum.

Maylor, E. A., \& Hockey, R. (1987). Effects of repetition on the facilitatory and inhibitory components of orienting in visual space. Neuropsychologia, 25, 41-54.

Miniussi, C., Wilding, E. L., Coull, J. T., \& Nobre, A. C. (1999). Orienting attention in time: modulation of brain potentials. Brain, 122, 1507-1518.

Neumann, O. (1987). Beyond capacity: a functional view of attention. In: H. Heuer, \& A.F. Sanders (Eds.), Perspectives on perception and action. Hillsdale, NJ: Lawrence Erlbaum Associates Inc. 
Nicoletti, R., \& Umiltà, C. (1989). Splitting visual space with attention. Journal of Experimental Psychology: Human Perception and Performance, 15, 164-169.

Nicoletti, R., \& Umiltà, C. (1994). Attention shifts produce spatial stimulus codes. Psychological Research, 56, 144-150.

Notebaert, W., Soetens, E., \& Melis, A. (2001). Sequential analysis of a Simon task-evidence for an attentional shift account. Psychological Research, 65, 170-184.

Posner, M. I. (1980). Orienting of attention. Quarterly Journal of Experimental Psychology, 32, 2-25.

Posner, M. I., \& Cohen, Y. (1984). Components of visual orienting. In: H. Bouma, \& D. Bowhuis (Eds.), Attention and performance $X$ (pp. 531-556). Hillsdale, NJ: Erlbaum.

Praamstra, P., \& Plat, F. M. (2001). Failed suppression of direct visuomotor activation in Parkinson's disease. Journal of Cognitive Neuroscience, 13, 31-43.

Proctor, R. W., Lu, C. (1994). Referential coding and attentional-shifting accounts for the Simon effect. Psychological Research, 56, 185-195.

Proctor, R. W., Lu, C.-H., Van Zandt, T. (1992). Enhancement of the Simon effect by response precuing. Acta Psychologica, 81, 53-74.

Rizzolatti, G., Riggio, L., Dascola, I., \& Umiltà, C. (1987). Reorienting attention across the horizontal and vertical meridians: evidence in favor of a premotor theory of attention. Neuropsychologia, 25, 31-46.

Rubichi, S., Nicoletti, R., Iani, C., \& Umiltà, C. (1997). The Simon effect occurs relative to the direction of an attentional shift. Journal of Experimental Psychology: Human Perception and Performance, 23, 1353-1364.

Schmitt, M., Postma, A., \& De Haan, E. (2000). Interactions between exogenous auditory and visual spatial attention. Quarterly Journal of Experimental Psychology, 53, 105-130.

Simon, J. R. (1969). Reaction toward the source of stimulation. Journal of Experimental Psychology, 81, 1974-1976.

Simon, J. R. (1990). The effects of an irrelevant directional cue on human information processing. In: R. W. Proctor, \& T. G. Reeve (Eds.), Stimulus-response compatibility: An integrated perspective. Amsterdam: North Holland.

Simon, J. R., \& Rudell, A. P. (1967). Auditory S-R compatibility: The effect of an irrelevant cue on information processing. Journal of Applied Psychology, 61, 354-358.

Simon, J. R., \& Craft, J. L. (1970). Effects of an irrelevant auditory stimulus on visual choice reaction time. Journal of Experimental Psychology, 86, 272-274.

Spence, C., \& Driver, J. (1997). Audiovisual links in exogenous covert spatial orienting. Perception and psychophysics, 59, 1-22.

Stoffer, T. H. (1991). Attentional focusing and spatial stimulus-response compatibility. Psychological Research, 53, 127-135.

Stoffer, T. H., \& Yakin, A. R. (1994). The functional role of attention for spatial coding in the Simon effect. Psychological Research, $56,151-162$.

Stoffer, T. H., \& Umiltà, C. (1997). Spatial stimulus coding and the focus of attention in S-R compatibility, the Simon effect. In B. Hommel, \& W. Prinz (Eds.), Theoretical issues in stimulus-response compatibility (pp. 181-208). Amsterdam: Elsevier.

Taylor, T. L., \& Klein, R. M. (1998). On the causes and effects of inhibition of return. Psychonomic Bulletin and Review, 5, 625-643.

Umiltà, C., \& Liotti, M. (1987). Egocentric and relative spatial codes in S-R compatibility. Psychological Research, 49, 81-90.

Umiltà, C., \& Nicoletti, R. (1992). An integrated model of the Simon effect. In J. Alegria, D. Holender, J. Junca de Morais, \& M.
Radeau (Eds.), Analytic approaches to human cognition. Amsterdam: North Holland.

Van der Heijden, A. H. C. (1992). Selective attention in vision. London: Routledge.

Van der Lubbe, R. H. J., Keuss, P. J. G., \& Stoffels, E.-J. (1996). Threefold effect of peripheral precues: alertness, orienting, and response tendencies. Acta Psychologica, 94, 319-337.

Van der Lubbe, R. H. J., \& Woestenburg, J. C. (1999). The influence of peripheral precues on the tendency to react towards a lateral relevant stimulus with multiple-item arrays. Biological Psychology, 51, 1-21.

Van der Lubbe, R. H. J., Wauschkuhn, B., Wascher, E., Niehoff, T., Kömpf, D., \& Verleger, R. (2000). Lateralized EEG components with direction information for the preparation of saccades versus finger movements. Experimental Brain Research, 132, 163-178.

Van der Lubbe, R. H. J., Jaśkowski, P., Wauschkuhn, B., \& Verleger, R. (2001). Influence of time pressure in a simple response task, a choice-by-location task, and the Simon task. Journal of Psychophysiology, 15, 241-255.

Van der Lubbe, R. H. J., Los, S. A., Jaśkowski, P., \& Verleger, R. (2004). Being prepared on time: On the importance of the previous and the current foreperiod on preparation, reflected in eventrelated brain potentials. Acta Psychologica, 116, 245-262.

Van der Lubbe, R. H. J., \& Postma, A. (2005). Interruption from irrelevant auditory and visual onsets even when attention is in a focused state. Experimental Brain Research, 164, 464-471.

Van der Lubbe, R. H. J., Jaśkowski, P., \& Verleger, R. (2005). Mechanisms underlying spatial coding in a multiple-item Simon task. Psychological Research, 69, 179-190.

Van der Lubbe, R. H. J., \& Van der Helden, J. (2006). Failure of the extended contingent attentional capture account in multimodal settings. Advances in Cognitive Psychology, 2, 255-267.

Van der Lubbe, R. H. J., Havik, M. M., Bekker, E. M., \& Postma, A. (2006a). Task-dependent exogenous cuing effects depend on cue modality. Psychophysiology. 43, 145-169.

Van der Lubbe, R. H. J., Neggers, S. F. W., Verleger, R., \& Kenemans, J. L. (2006b). Spatiotemporal overlap between brain activation related to saccade preparation and attentional orienting. Brain Research, 1072, 133-152.

Verfaellie, M., Bowers, D., \& Heilman, K. (1988a). Attentional factors in the occurence of stimulus-response compatibility effects. Neuropsychologia, 26, 435-444.

Verfaellie, M., Bowers, D., \& Heilman, K. (1988b). Hemispheric asymmetries in mediating intention, but not selective attention. Neuropsychologia, 26, 521-531.

Verfaellie, M., Bowers, D., \& Heilman, K. M. (1990). Attentional processes in spatial stimulus-response compatibility. In: R. W. Proctor, \& T. G. Reeve (Eds.), Stimulus-response compatibility: An integrated perspective. Amsterdam: North Holland.

Wallace, R. J. (1971). S-R compatibility and the idea of a response code. Journal of Experimental Psychology, 88, 354-360.

Wascher, E., \& Wolber, M. (2004). Attentional and imtentional cueing in a Simon task: An EEG-based approach. Psychological Research, 68, 18-30.

Wascher, E., \& Wauschkuhn, B. (1996). The interaction of stimulusand response-related processes measured by event-related lateralizations of the EEG. Electroencephalography and Clinical Neurophysiology, 99, 149-162.

Zimba, L. D., \& Brito, C. F. (1995). Attention precuing and Simon effects: a test of the attention-coding account of the Simon effect. Psychological Research, 58, 102-118. 